which screws on and closes it. Messrs. Maw, Son, and Thompson of Aldersgate-street make the apparatus.

Under the form of acetic acid and ferrocyanide of potassium, the ferrocyanic test has for a long time been favourably regarded as a delicate test for albumen. Senator, in his recently published monograph on albuminuria, in referring to the drawbacks belonging to heat and nitric acid, speaks thus ${ }^{1}$ of the test:- "As, however, notwithstanding these drawbacks, the test in question [heat and nitric or acetic acid] is the only one in common use, it will not be superfluous to give a brief account of far more reliable tests which should be used in doubtful cases, or I would rather say, in all cases in future. These are as follows: (1) To acidify the urine with acetic acid and then add carefully a (concentrated) solution of ferrocyanide of potassium, according to Hofmeister the most delicate of all the tests for albumen, and which precipitates all the albuminuus bodies, but not peptone." To adapt the ferrocyanic test conveniently for clinical use, it occurred to me to follow what I had already done as regards the cupric test for sugar, and bring it into the pellet form. At first I placed the acid (citric) and ferrocyanide in the same pellet, and these pellets were introduced to the notice of the profession at the Clinical Society in the early part of 1883. Subsequently, for reasons that presented themselves as experience was gained with the employment of the test, the agents were placed in separate pellets, and it is in this form

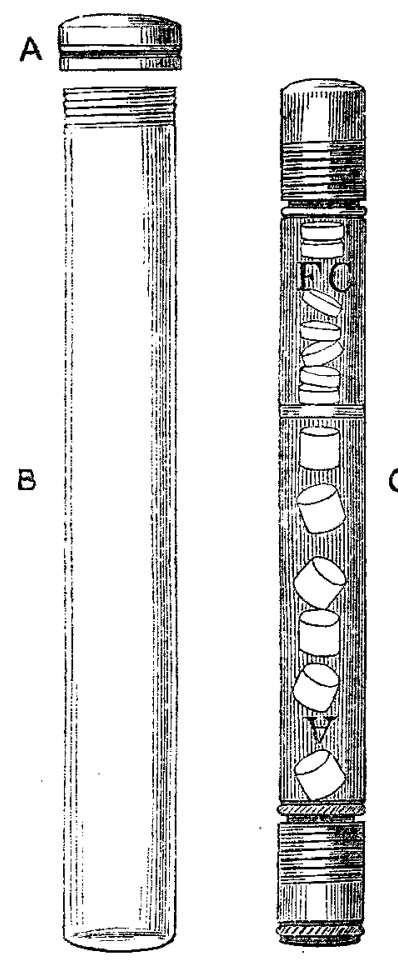
that I now recommend it. As the pellets are devoid of any foreign principle whatever, there is nothing to undergo deterioration by keeping. Citric acid is just as eligible for the purpose required as acetic acid. Enough is supplied in the pellet to secure the presence of free acid in any alkaline specimen of urine that is likely to be come across, provided the quantity mentioned in the instructions for the application $c$ of the test is taken. Should any doubt exist, two pellets can be used in place of one. The ferrocyanide pellet consists of the sodium instead of the potassium salt, on account of its yielding a looser and more speedily soluble product. The pellets are made by $\mathrm{Mr}$. Cooper of 66, Oxford-street.

In using the test about a drachm of urine is taken as the quantity to be employed. An acid pellet is dropped into it, and with a little agitation is found to be quickly dissolved. One of the ferrocyanide pellets is next dropped in, and the urine again shaken to facilitate solution. If albumen is present, a precipitate immediately appears.

It is necessary that the test should be used in the manner which has been mentioned-that is, the acid pellet must be first dropped in and dissolved, and then the ferrocyanide pellet added. The latter pellet it is which supplies the information required, and if a precipitate is by this pellet produced, the conclusion may be drawn that albumen is present, for the test does not precipitate peptones or other principles incidentally present in the urine. For sensitiveness the ferrocyanic will bear comparison with any other albumen test; and should no precipitate be rendered visible it may be safely inferred that no albumen is present. There is no need therefore for anything beyond the test itself being employed, and no spirit lamp being required, gives it a great advantage.

It occasionally happens that a precipitate is produced by the acid pellet alone, but no fallacy should arise from this. It is the ferrocyanide pellet which affords the evidence of the presence or absence of albumen. The acid pellet only supplies a preparatory condition to allow the ferrocyanide to act, and does not constitute the test. The usual source of the precipitate referred to is uric acid, and by diluting the urine with about an equal bulk of water, it is prevented rom appearing, In this way the interference thus occasioned to the application of the test is removed. Mucin also is precipitated by an acid, and the best plan here is to take two test tubes and to treat the urine in one mitir the acid pellet alone, and in the other with both pellets. A com parison of the two will show whether albumen is present or not.

Should the urine be turbid from lithates, it may be cleared either by the application of moderate warmth, or, what may be more convenient, the addition of about an equal bulk of hot water.

A most portable little case is made by Jahncke of Cannnbury Works, Dorset-street, Essex-road, N., which contain. the two kinds of pellets for albumen, the cupric test pellet for sugar, a spirit lamp, test tube, and urinometer. This gires all that is required for the ordinary examination of the urine and makes an elegant, compact, and convenient little case.

Grosvenor-street, W.

\section{THE ETIOLOGY OF GOITRE IN ENGLAND}

\section{BY W. N. THLRSFIELD, M.D.}

As a rule the subjects which are brought for discussion before the Society of Medical Officers of Health relate either to matters connected with sanitary administration, or to infectious disease, or to influences acknowledged to directly affect the health of the community. As goitre may not at first sight appear to belong to either of these classes, it may be as well for me to state that my attention was first directly drawn to this subject about three years ago, in consequence of $\mathrm{my}$ being requested by a sanitary authority to inquire into an epidemic prevalence of goitre amongst the children in some schools in their district. The undoubted epidemic nature of the disease and other facts were so diametrically opposed to the etiological views almost universally given in the text-books on medicine, that I was induced to make further investigations as to the general liabilities of the population and physical characteristics of certain districts where goitre was endemic, and, in order to avoid hasty generalisation from general conditions, which may have been merely coincidences, to check these observations by particular inquiries in individual cases, wherever opportunity offered.

I have restricted my conclusions as to the cause of goitre to the disease as it occurs in England, because I wish to confine myself as far as practicable to personal experience and there may be in other countries contributing causes of which 1 have no knowlerige. It would, for instance, be interesting to know why in India goitre should be nearly as common in men as in women.

For a definition of goitre I do not think I can do better than follow the official nomenclature of the Royal College of Physicians, which defines goitre as "enlargement of the thyroid gland, endemic in certain mountainous districts, but not limited to them." This enlargement depends upon simple expansion, hypertrophy of tissue, or vesicular distension, or a combination of all three, and results from persistent abnormal increase in the blood in the gland, or persistent abnormal call upon the physical action of the gland, and usually upon both combined. Goitre is much more prevalent amongst females than males, and certainl in my experience affects in undue proportion the industrial class. The primary causes of goitre are as follows (1) Climatic, (2) physiological (including sex, heredity, \&c.), (3) industrial, (1) dietetic, (5) neurotic and strumous diathesis.

In localities where goitre is endemic two of the abore causes are always present, and generally three or four, in various degrees of preponderance, and the preponderating cause will often be found to differ in different localities Sporadic cases of goitre occurring in localities where goitre is not endemic, when not directly imported, are the result of the preponderating influence of some one or two of the above causes, probably conjoined in many instances with the influence of heredity. The causes are mentioned in the order it will be most convenient to consider them. I hare not referred to absence of sun light, confined atmosphere. and other causes considered as contributing in some

1 Paper read before tha Snitety of Medical Officers of Halth May 1ath, 188.5. 
countries, as these are not in my experience concerned in producing English goitre.

The Climatic Influence. - This is as a rule the chief factor in the production of endemic goitre. Endemic goitre may elsewhere exist in low-lying plains, due doubtless to the combination of some of the causes above alluded to; but in England, so far as my experience goes, the endemic prevalence of goitre is not found at much less altitude than is indicated by a fall in the column of mercury equal to about three-quarters of an inch. This diminished atmospheric pressure in hilly districts, together with the climbing of hills and other conditions leading to sudden alterations in the circulation, combined with the natural distensile property of the thyroid, is, I believe, the origin of the goitrous diathesis. In the goitrous districts abnormal enlargement of the thyroid occurs at any age up to adult life. At the present moment I could show cases at almost any age from infancy upwards. It appears, however, usually to show itself from five to twelve years of age, perhaps most commonly at about eight years of age; but where medical advice is sought, it is not generally until a marked enlargement coincides with the first catamenial period or some other cause interfering with the circulation. It is this circumstance, I think, which has given rise to the statement found in most text-books that it is at the above period that goitre usually makes its first appearance. It is my experience that a much larger proportion of cases of enlargement of the thyroid in a goitrous district will be found in females under the age of thirteen than above it. The enlargement in the majority of cases then disappears; in some, however, it is persistent and increases much, and it is these cases which are ordinarily known as goitre. This persistence is owing to causes specially interfering with the circulation of the thyroid or otherwise conducive to hypertrophy of the gland. The cause most directly conducive is, however, diminished pressure of the atmosphere.

The Physiological Infuence.-Of the thyroid gland little is definitely known beyond its anatomical structure, which is that of a very vascular ductless gland, with a dual supply of bloodvessels and nerves from different sources. The thyroid has this peculiarity, that of all the glands of the body which we can see and feel it is most prone and most capable of alteration in volume, and it is to this peculiarity that goitre is due. It is, in fact, the giving way under varying degrees of increased force below, or diminished pressure above of the point of least resistance in the human body externally. In other words, the abnormal and persistent expansion of a structure normally distensile for physiological reasons. I have no personal information on the point, but it is stated that goitrous tumours increase in size in wet weather-i.e., with a low barometer; and a locality with a high local humidity might in the same way be a contributing influence. The function of the thyroid in the human body appears to be of a double nature-one almost mechanical the other physiological. One purpose served by the th yroid appears to be to act as a diverticulum for the circulation on the occurrence of arterial tension in the brain or uterus. That it should so act in the case of the intercranial circulation might be anticipated from the very intimate anatomical relation between the arterial supply to both, and cases have been recorded of acute goitre supervening on intense excitement. This function or habit of the thyroid has much to do, as will be presently shown, in the production of goitre. That it does so act in the case of the uterus is also an observed fact, sudden enlargement of the thyroid frequently supervening in connexion with menstruation and pregnancy, and in some cases a periodical expansion occurs. As no direct anatomical connexion between the uterus and the thyroid is traceable, we are driven to the explanation of correlation of nerve influence between the two organs. That intimate sympathy exists between the generative organs and those of the throat is obvious from well-known facts; and the liability of sudden enlargement of the thyroid to follow uterine excitement appears to have been utilised for practical purposes both in classical and mediæval times. This function or habit of the thyroid would account for the greater liability of the female to suffer from enlarged thyroid. The thyroid is at all ages normally proportionately larger in the female than in the male; and the fact, as I have observed, that the preponderance of cases of enlarged thyroid amongst females is found, though perhaps not to the same extent, in childhood as in adult life, is evidence that this special liability is not, as has been suggested, owing to the greater use of water as a beverage by females, but is a liability of the sex, and doubtless the result of a natural provision for equalising the sudden alterations in the circulation to which females are specially liable.

As regards the function of the glandular apparatus of the thyroid (the vesicles, \&c.), little is known definitely. Physiologists seem generally to agree that the gland performs some office in connexion with the composition of the blood. Dr. Baber frequently found the vesicles to contain red blood-corpuscles in various stages of degeneration and decolourisation, and concluded that the gland was concerned in the retro-transformation and excretion of the elements of the red blood-corpuscles. This assumption, if correct, has a most important bearing, and may help to throw light upon the mysterious but undoubted occasional connexion between the use of certain drinking waters and the production of goitre, and will be referred to subsequently in connexion with that point. Goitre has a most decidedly hereditary tendency. I have seen many such cases both direct and collateral. It has been suggested by Dr. Woakes, on analogical grounds, that this hereditary tendency would be most marked where there was a strumous taint, inducing goitre by abnormal relaxation of the arteries of the gland, the result of neurosis of the cervical ganglia. The cases, however, in which I have noticed heredity have generally been of a high physical standard of development, and of an average intellectual standard.

The Industrial Influence. - That goitre is frequently entitled to be considered an industrial disease I entertain no manner of doubt whatever. Carrying weights on the head or otherwise, so as to interfere with the cerebral circulation, is a most potent contributing cause. It requires, however, to be combined with the climatic or some of the other influences alluded to, and even in goitrous districts I have obtained strong evidence that for the industrial influence to be operative it is necessary as a rule that it should commence at or soon after puberty. I am well acquainted with two populous localities both situated at an altitude of about seven hundred feet. In both goitre used to be endemic, and is so to some extent at the present time. In both the watersupply is the same as it has been from time immemorial; in both there has been a most marked diminution in the number of cases of goitre, especially of the more severe kinds, and the only alteration in the habits or liabilities of the inhabitants which could at all affect this has been the cessation on the part of the young females of the locality of the habit of carrying weights on the head in their employment in connexion with the coal and ironstone mines, also their daily water-supply, \&c.; as having acquired the ability to readily carry weights on their heads, they adopted that mode of carrying everything. The present generation of females in the locality, not having had a similar education, carry water \&c. in the ordinary way. Of one of these localities I have intimate knowledge, and well remember as a boy passing through the streets to have frequently noticed the repulsive looking huge goitrous tumours of a kind one seldom, if ever, sees now. At the present time the alteration is most marked and is at once admitted by the older inhabitants. This district is a mining and manufacturing one, more flourishing years ago than now, and situated close to a navigable river, and no explanation of greater mixing of population and cessation of intermarriage would apply here. The only alteration here has been industrial. Years ago this locality was so notorious for goitre as to give a name to the affection in that part of England. The women were also celebrated for their ability to steadily carry great weights on their heads, and at certain seasons of the year many of them were engaged to go to other localities and occupations where such an accomplishment was desirable. Following up this clue, I have found several instances of sporadic goitre where the only member of the family affected with goitre has been the only one who had carried weights on the head habitually; these hare generally been people dwelling at a considerable altitude. In carrying weights on the head the return circulation of the neck is retarded and the arterial stimulated, conditions directly tending to congestion of the thyroid, and this I believe to be the most potent industrial influence, but any heavy exertion has a tendency to the same effect; and this would probably explain the not unfrequent cases in which domestic servants develop goitre in non-goitrous districts. In these cases there would, I think, generally be present hereditary tendency to goitre or a strumous and neurotic diathesis. I hare ascertained that the traditional practice of one eminent 
medical man who practised in a goitrous district in the midlands before the introduction of iodine was to strictly enjoin upon his goitrous patients the necessity of not carrying weights on their heads and to boil the drinking water. This boiling of the water was probably intended to get rid of some of the lime salts, but it would also precipitate the iron where lime was present.

The Dietetic Infuence.-If in my investigation into the origin of goitre $I$ could have made my mind a complete tabula rasa as to all that I had previously heard or read on the subject, I do not tnink I should have found it necessary to consider the probable influence of the water at all in the matter. I am, however, disposed to think that in some of the localities water was certainly a contributing cause. In each of these cases a theory of excess of saline and earthy matters in solution, or of iron in solution in small quantities, would have been equally applicable. I have adopted the theory of iron, as it appears to me that it can be more readily and scientifically connected with hyperæmia of the thyroid body than excess of dissolved matter in the water consumed. In looking through the various text-books of medicine, nothing is more striking than the uniformity with which the quality of drinking water is assigned as the cause of goitre, though on the question of the element in water producing the evil the diversity of opinion is almost as remarkable. Personally 1 venture to express a belief that water is by no means essential to the production of the disease, and has frequently nothing to do with it. That every kind of water from snow-water downwards has been credited with producing the disease is presumptive evidence that water in some instances has had nothing to do with it, and I am satisfied that under favourable conditions of climate and industry goitre may occur altogether independently of the water, and in the epidemic above alluded to, the water-supply of the majority of cases was derived from the hard Cambrian formation, and to quote the report of the well-known analyst, Mr. Sutton of Norwich, was " one of the purest natural waters which had ever come under his notice or of which he had seen any record." It did, however, contain slight traces of iron. Indeed, the very fact of the existence of an epidemic is, in the case of such a disease as goitre, strong evidence that water had nothing to do with the malady. Water has, however, been credited as the cause of goitre from the earliest records of medicine, and there is abundant testimony that it is often a potent contributing cause of the affection. To my mind no satisfactory explanation as to how it acts has yet been afforded, and on this point I speak with special diffidence. The theory I have to offer in the explanation of the goitriferous property of certain waters has however, I believe, sufficient basis of fact and probability to justify my bringing it before you. of the numerous hypotheses which have been brought forward to explain the influence of water in the production of goitre, the two which have received most support from observers on the subject, and which appear to have the broadest basis of the fact are (1) the theory that goitre follows the use of water from magnesian and limestone rocks, and (2) the theory that the presence of iron pyrites in the strata from which the water is drawn is the cause. I believe that in the coincidence of these two may be found the true explanation, or, to speak more accurately, that the presence of iron in the strata in a form liable to pass into a soluble salt of iron on meeting with the ordinary spring water of the district may account for the above discrepancies, and afford a rational explanation. I am quite aware of the objection to the above theory, that iron is very universally diffused and constantly occurs in water where no goitre is found. This would, however, apply with equal force to any of the other properties of water which have been suggested as the cause. Iron pyrites is insoluble, but when brought in contact with spring water containing carbonate of lime and magnesia in carbonic solution it gradually passes by a process of exidation into cárbonate of iron. 'This salt of iron is, according to Professor Ringer, most readily and directly assimilated by the system. Now, the mode in which the water tends to aggravate or promote goitre may possibly be as follows: Iron is in a certain sense food, and is necessary and conducive to the formation of the red corpuscles of the blood. Administration of iron in anæmia increases the number of the red bloodcorpuscles and the amount of iron contained in them, and although such result may not take place in health, it is not too much to assume that a readily assimilated form of iron dissolved in minute quantities in water would act as food for and stimulate the production of the red corpuscles, and so throw more work upon the glands which deal with these corpuscles in the system. Yow, the most recent view as to the physiological function of the thyroid appears to be that it is an excretory organ for the effete blood-corpuscles, and may it not be that the observed effect is the result of undue ingestion of that form of food with the excretion of which the gland is concerned. I use the word food advisedly, because I believe (assuming the theory to be correct) the result to be due not to the ingestion of large quantities of iron, but to the habitual and constant consumption of minute traces of iron in a most readily assimilable form, and which would pass into the system as albuminate of iron. The carbonate of iron indted under such conditions in natural water would be constantly in a state of change, and would only exist. in the water in small amount in ordinary circumstances, and imperceptible to taste, and being a most unstable compound it is readily precipitated. This is a reason why iron in this form is not unfrequently overlooked, as after the lapse of some time and a little exposure the iron is precipitated, and no reaction follows the tests for iron applied to water in ordinary sanitary analysis. The therapeutic effects of iron may throw some light upon this point. When iron is administered therapeutically, a sensation of fulness and pain in the head sometimes supervenes, and is recognised as indicating the desirability of discontinuing the medicine. This would give support to the theory advanced. On the other hand, anæmia is frequently present with 'goitre. This may be due to the blood-elaborating function of the thrroid being out of order; but iron is said, in some cases of anæmia accompanied with goitre, to produce marked benefit. I believe these cases will be found where a strumous and neurotic diathesis is the preponderating cause. In connexion with this subject, I think it well to refer to the instance so frequently quoted of French peasants, who are alleged, in order to avoid the conscription, to artificially produce goitre by drinking goitrifernus water. According to the best account I can obtain, the conscripts are said to "go up to Briançon." Briançon is, I believe, upwards of four thousand feet above the sea, and this fact, combined with the goitrous diathesis of many Frenchimen, would be powerful contributing influences.

Is Goitre diminishing in England?-Endemic goitre certainly is. In a disease where the influence of heredity is 80 marked, we may assume that the endemic prevalence may to some extent in isolated communities have been due to intermarriage, and that increased migration and facilities for travel would certainly tend to produce a decrease; but I am persuaded that the chief cause is due to the disuse of the practice of carrying weights on the head. This would chiefly apply to those hilly districts where a slight contributing influence, in addition to that of diminished atmospheric pressure, is all that is needed to induce the deformity. Throughout Fngland generally there has been, during the last quarter of a century, a marked alteration in the custom of the rural population in the matter of carrying weights. To carry weights on the head safely, and with comfort, requires early training and practice. Before the introduction of tins with handles, the wooden milk pails with side handles were of such a shape and make that when full they could not well be carried by one person except on the head. Hence the early training and acquired facility in carrying weights on the head. The introduction of tin ware with handles has altered this, and led to the disuse of the practice, and, as I believe, may thus be said to have contributed to the diminution of goitre in some agricultural districts in England. With sporadic goitre in towns the case may be different This affection is said by some to be on the increase. If this be so, and, considering the element of debility present in so many of these cases, the neurotic influence may be the preponderating one, and the theory advanced by Dr. Woakes is probably correct-viz, that this affection shares in a general increase of neurotic affections in the present age.

Epidemic Goitre.-Time will not allow me to do more than to refer to the epidemic of goitre mentioned in the commencement of this paper. It had been noticed for some months before $I$ investigated it, and the cause $I$ beliere to have been purely climatic, probably aggravated by some meteorological condition peculiar to the year.

Connerion of Goitve with Cretinism.-On the theory of goitre being a circulatory disease and dependent to a large extent on atmospheric influences, the connexion of cretinism with goitre seems to admit of explanation. In some countries the goitriferous influences, including the strumous 
diathesis, may be sufficient to produce goitre and cretinism in the same individual. In this country cretins are rare, and in the majority of such cases in which a post-mortem examination has been obtainable, the thyroid has been found absent. Now, assume an infant of a strumous diathesis with deficient arterial tone under strong goitriferous influences. In the absence of the safety valve action of the thyroid, a cretinous condition would be expected to result. Indeed, the converse may be true, and I have recently met with a microcephalic idiot with a huge goitre.

\section{NOTES ON TWO CASES OF REMOVAL OF THE JAW FOR TUMOURS.}

\section{BY F. F. PERRY,}

SURGEON, BENGAI MEDICAL SERVICE ; LATE DEMOXSTRATOR OF ANATOMY, WESTMINSTER HOSPITAL.

Trre cases to which the notes below refer occurred during the present year and exhibit several points of interest.

CASE 1. Large naso-pharyngeal polypus, removed by displacement of the superior maxilla.-Badawah, Mahomedan, male, aged forty, was admitied into the Mayo Hospital, Lahore, on April 26th, 1884. His general health tairly good. Nothing noteworthy regarding his family, habits, \&c. His present illness commenced three years ago, when he noticed some obstruction in his right nostril. Since then the obstruction has progressed gradually with swelling of the right cheek, difficulty in breathing and swallowing, and a moderate amount of pain. Urgent symptoms, connected with dyspnœa, dysphagia, and want of rest, have been present during the past three or four months, and lately he has lost much flesh.

Present state.-A thin and weakly-looking man, evidently considerably emaciated and with a marked look of distress; breathes with mouth wide open, and voice has a marked nasal twang. The right cheek is seen to be bulged outwards en masse, the outline of the bone not being interfered with. At the inner angle of the right eye is a soft swelling, the shape and size of an almond, which can be emptied of thick mucoid material into the nose by applying pressure over it. Both nostrils are completely blocked, the right by a soft reddish growth which extends to within a quarter of an inch of the orum nasi, and the left by the septum, which is pushed over in a very marked degree. On looking into the mouth the hard palate is seen to be natural, but the whole of the upper two-thirds of the pharynx is occupied by a rounded mass which pushes the soft palate down in front of it, so as to interfere considerably with respiration. Marked dysphagia is also present, and liquid food only can be taken. The diagnosis of naso-pharyngeal polypus of large size and probably high attachment was made. The patient's general condition was good, and he was very anxious for something to be done.

On April 28th chloroform gas was given by the mouth, but produced troublesome symptoms owing to pharyngeal obstruction. Tracheotomy was performed and the inhalation of chloroform carried on without further hitch through the tube. The right superior maxilla was then displaced in the ordinary way, the superficial incision being carried through the centre of the upper lip, along the side of the nose and lower margin of the orbit, and the flap turned downwards. The bone was then divided by saw and bone forceps and displaced outwards, the soft palate being separated transversely from the hard on the right side. An enormous soft reddish growth was found occupying the nasopharyngeal and right nasal cavities, having three or four points of attachment to the under surface of the skull. The largest portion (about the size of a medium orange) was detached and removed with difficulty through the mouth. Various smaller pieces were torn off and withdrawn from in front and the stump freely cauterised with the hot iron. Throughout the operation there was remarkable freedom from hæmorrhage. The bone was then replaced and kept in situ by drilling the malar process and wiring it together with silver wire and connecting the incisor teeth in the same manner. The flap was sutured with alternating wire and catgut sutures, the soft palate being left untouched.

The man was placed in a separate ward, which was kept at a uniform temperature and carefully steamed at night. The tube was left in the trachea for the first twelve hours, and covered with a light bag of pine sawdust, purified with eucalyptol. At the end of this time it was removed, and a light bandage applied. Recovery was uninterrupted, and very rapid. The patient slept well and could take large quantities of liquid nourishment. Pulse of good quality, and the temperature never exceeded $1005^{\circ}$. On the third day his condition was as follows:-Temperature $100^{\circ}$; pulse 90 ; wound entirely and soundly healed by first intention, with the exception of a small opening through the soft palate, and another at the point of exit of the wire through the malar bone; tracheal opening contracted to the size of a crowquill; moderate amount of inflammatory swelling about the right side of the face, but this rapidly disappeared. All the sutures were removed, and on the seventh day the man sat up in bed and ate soft solid food, being quite free from all symptoms of dyspnoea and dysphagia. The wire suture was removed on the tenth day, and in the following night he considered himself well enough to run away, a common practice with patients in this country. He was seen at work accidentally about a week afterwards.

Remarks.-The points of interest in connexion with this case are: 1. The large size of polypus. 2. The great help obtained during the operation by first performing tracheotomy. 3. The rapid recovery of the patient.

Case 2. Entire excision of the lower jaw for myeloid sarcoma.-Mussamat Jhandi, a female Hindoo aged thirtytwo, was admitted into Jullunder Dispensary on September 26 th, 1884.

Her general health had been good; mother of several children, and is at present nursing one. Has had occasional attacks of intermittent fever, but not seriously. No history of syphilis. Her present illness commenced two years ago with a swelling about the central incisors of the lower jaw; very gradual at first, but much more rapid during the last three months. Has emaciated considerably of late, and especially since the protrusion of the growth between the lips (about two months). Can assign no cause, and has not had much pain, but suffers principally from the inconvenience arising from the position of the growth and the difficulty in masticating and speaking.

Present state.-A slightly made and much emaciated woman about thirty years of age. Temperature normal; pulse weak and small; respiration healthy. No disease present, with the exception of that in the lower jaw, which is the seat of a growth of a reddish colour, part of which protrudes between the lips. The protruded portion is the size of a large walnut, irregularly lobulated and ulcerated on the surface from constant exposure to air and dirt. The lips cannot be made to close over it. Internally the growth is found to involve the lower jaw, principally at its upper or dental margin, and to be of a soft, almost fungating nature, with the teeth embedded and lying quite loosely in it. The bone itself is felt to be considerably expanded in its upper half, the limits being on the right side beyond the last molar tooth, and on, up to, and including the first molar. The lower border of the bone is free, but behind the symphysis there is a nodule, about the shape and size of half a walnut, which appears to be springing from the bone. The whole growth is about the size of a small orange, and gives the jaw a peculiar pointed appearance. No enlargement of glands can be detected, nor are the superficial structures in any way involved. There is the greatest difficulty in eating anything, even liquids being taken with considerable effort, so that really the woman is being slowly starved. Speech is so much interfered with that her statement is made out with difficulty. She is extremely anxious for an operation to be performed.

She was kept quiet in hospital, and fed up as far as possible for twelve days, taking also small doses of quinine. During this time it was noticed that there was a decided increase in the size of the growth and its protrusion from the mouth.

Operation.-On Oct. 9th, under chloroform, laryngotomy was performed as a preliminary step in order to facilitate the main operation by obviating the risk of the respiration being impeded by the falling back of the tongue over the glottis, and also to facilitate the administration of chloroform. A ligature was then passed through the tip of the tongue, to retain command over it in case of any accident to the tube in the larynx. At this stage, owing to the failure of the heart's action, the pulse, which had been very bad throughout, ceased altogether, and artificial respiration 